\title{
Neoadjuvant therapy in urothelial cancer
}

\author{
New therapies on the horizon
}

Stephan Brönimann · David D'Andrea · Shahrokh F. Shariat · Kilian M. Gust

Received: 5 August 2019 / Accepted: 4 October 2019 / Published online: 23 October 2019

(C) The Author(s) 2019

\begin{abstract}
Summary Neoadjuvant cisplatin-based chemotherapy is standard treatment for muscle-invasive bladder cancer before radical cystectomy (RC). Despite level 1 evidence demonstrating an overall survival benefit for patients undergoing RC after neoadjuvant chemotherapy (NAC), acceptance rates are still low. In high-risk upper tract urothelial cancer (UTUC), cumulative evidence suggests that NAC for locally advanced UTUC can improve oncological outcome. Ongoing phase 3 trials will finally prove the benefit or futility of NAC in this tumor entity. Since urothelial cancer (UC) is a heterogeneous disease, predictive biomarkers are needed to select specific patient populations and potentially increase response rates to NAC. Novel targeting therapies, including immune checkpoint inhibitors, have been approved for metastatic UC. In combination with predictive biomarkers, these might have the potential to change systemic therapy for UC from a "one-fits-all" principle to a more individualized approach.
\end{abstract}

\footnotetext{
S. Brönimann · D. D’Andrea · S. F. Shariat .

K. M. Gust, MD $(\bowtie)$

Department of Urology, Comprehensive Cancer Center,

Medical University of Vienna, Waehringer Guertel

18-20, 1090 Vienna, Austria

kilian.gust@meduniwien.ac.at

\section{S. F. Shariat}

Department of Urology, Weill Cornell Medical College, New York, NY, USA

Department of Urology, University of Texas Southwestern, Dallas, TX, USA

Department of Urology, Second Faculty of Medicine, Charles University, Prague, Czech Republic

Institute for Urology and Reproductive Health,

I.M. Sechenov First Moscow State Medical University,

Moscow, Russian Federation
}

Keywords Urothelial cancer · Neoadjuvant therapy · Chemotherapy · Targeted therapy · Immune checkpoint inhibition

\section{Take home message}

- Neoadjuvant chemotherapy for muscle-invasive bladder cancer (MIBC) is the standard in cisplatineligible patients and should be given for MIBC prior to radical cystectomy.

- Neoadjuvant chemotherapy for high-risk upper tract urothelial cancer (UTUC) is still controversial and lacking level 1 evidence. Extrapolation from MIBC data and the high rate of renal insufficiency after radical nephroureterectomy resulting in cisplatin-ineligibility, make neoadjuvant chemotherapy (NAC) for high-risk UTUC a rational approach.

- Checkpoint inhibitors (CPI) and other targeted therapies in combination with development of predictive biomarkers have the potential to change neoadjuvant treatment strategies in patients with urothelial cancer and allow individual strategies for systemic therapies rather than the currently used "one-fits-all" principle.

\section{Introduction}

Approximately half of patients with muscle-invasive urothelial bladder cancer develop metastatic disease within two years after radical cystectomy (RC), resulting in a 5-year survival of roughly $50 \%$ [1-5]. Neoadjuvant administration of platinum-based combination chemotherapy has demonstrated a significant survival benefit compared to RC alone, leading to an improvement in 5-year overall survival (OS; $50 \%$ vs. $45 \%$; $13 \%$ reduction in risk of death) with an absolute difference in disease-free survival of 9\% [6-8]. Despite level 1 evidence and recommendation in international guide- 
lines, the use of neoadjuvant chemotherapy (NAC) prior to $\mathrm{RC}$ remains low.

Albeit, analysis of retrospective data does not show any difference between the currently used NAC regimens in terms of complete response and pathologic stage after RC $[8,9]$, they have not been compared in prospective randomized phase III trials; thus, the ideal NAC regimen has not been established yet.

\section{Overview of current treatment approach}

\section{Currently used different NAC regimens:}

- MVAC (methotrexate, vinblastine, doxorubicin, and cisplatin)

- Dose-dense MVAC (ddMVAC) with granulocyte colony-stimulating factor (G-CSF) support and double the dose-intensity of cisplatin and doxorubicin, while reducing the dose of methotrexate and vinblastine by one third compared to classic MVAC

- $G C$ (gemcitabine, cisplatin)

- $C M V$ (cisplatin, methotrexate, and vinblastine)

\section{Muscle-invasive urothelial bladder cancer}

MVAC is the best studied regimen in the neoadjuvant setting. SWOG-8710 demonstrated a benefit for NAC with MVAC plus RC over RC alone with a trend in beneficial median OS (77 vs. 46 months) and 5-year OS rate of 57 vs. $43 \%$ ( $p=0.06)$ [10].

There are no published prospective trials evaluating ddMVAC in the neoadjuvant setting, but retrospective data imply that this regimen seems to be a reasonable option. ddMVAC has been reported to have less toxicity than MVAC with lower rates of febrile neutropenia and treatment-related deaths, resulting in fewer delays of administration and a reduction in time to surgery [10-12].

As a result, this regimen has become more frequently used at some centers. Recently, Peyton et al. demonstrated superior downstaging rates for ddMVAC, over GC and gemcitabine/carboplatin with $52.2 \%, 41.3 \%$, and $27.0 \%$, respectively. Similar superiority was shown in terms of pathological complete response (pCR; ypT0pN0) with $41.3 \%, 24.5 \%$, and $9.4 \%$, respectively. However, the difference in OS did not reach statistical significance [13].

Extrapolating from the metastatic setting, where the combination of GC has comparable efficacy with better tolerability than MVAC, GC has become the most commonly used regimen in the neoadjuvant setting for muscle-invasive bladder cancer, despite the lack of level 1 evidence in this setting [8]. Despite no significant difference in pCR rates between MVAC and GC, Yin et al. noted, in a meta-analysis, a significantly reduced OS for GC, which was still inferior but not statistically significant after excluding patients who received carboplatin-based combination chemotherapy [14].
In summary, neoadjuvant cisplatin-based combination chemotherapy improves survival outcomes in muscle-invasive urothelial bladder cancer, but the optimal neoadjuvant regimen has not been established.

Additionally, NAC in urothelial cancer is still underutilized. According to the National Cancer Data Base (NCDB), only 19\% (1619/8732 patients) receive NAC before RC. Although these patients had significantly higher pT0 rates after NAC, despite being lower than stated in the INT-0080 trial (13 vs $38 \%$ ), the difference in pT0 rates did not translate into a clear survival advantage after adjusting for immortal-time bias.

Considering the fact that the NAC regimens used are not mentioned, these results have to be interpreted with caution. Diversity in clinical outcome might also be related to differences between patient populations included in clinical trials and the "real world scenario". Therefore, there is a medical need to define more precise selection criteria to determine who will benefit the most from neoadjuvant therapy $[10,15]$.

\section{Upper tract urothelial cancer}

Considering the substantial risk of becoming cisplatin-ineligible after radical nephroureterectomy, NAC should also be discussed in the context of highrisk upper tract urothelial cancer (UTUC). While several clinical trials are on the way [16], level 1 evidence for NAC in UTUC is still lacking. Matin et al. evaluated the incidence of pathologic downstaging and pCR in patients with high-grade UTUC. Significant downstaging in study group patients compared with the historic controls was described with $14 \%$ of patients showing a pathological CR [17].

Randomized phase 3 trials are ongoing, while experiencing difficulties in accrual of patients, in part, because of the rarity of the disease [18-20].

Despite the lack of level 1 evidence, the cumulative evidence suggests that NAC for locally advanced UTUC can improve oncological outcome. Nevertheless, prospective studies are needed to clarify the clinical benefit of NAC for patients with locally advanced UTUC, since there are divergent data on the significance of NAC in terms of OS benefit [21]. Clinical trials evaluating the use of neoadjuvant immune checkpoint inhibitors in high-risk UTUC are on their way [22] with the potential to increase the generally low acceptance of neoadjuvant therapy in urothelial cancer [23].

\section{Neoadjuvant immune-checkpoint inhibitors}

The success of immune-checkpoint inhibitors (CPI) in metastatic urothelial carcinoma led to their application in the neoadjuvant setting. Three prospective phase I/II studies, indeed, examined the efficacy and safety of CPI in bladder cancer patients [24-26]. 
Preliminary results of the ABACUS trial, a single arm study investigating two cycles of atezolizumab every three weeks in cisplatin-ineligible patients with muscle-invasive bladder cancer were presented at ESMO 2018. This trial includes 74 patients, of whom 59 received the planed two cycles of neoadjuvant atezolizumab. The pCR rate was $29 \%$ in all comers and was even higher in patients with PD-L1 positively tested tumors (40\%). Significant progression of the disease appears infrequent and the majority of patients $(67 / 74)$ underwent RC [25].

The PURE-01 trial, which included patients with histological confirmed urothelial carcinoma $\leq \mathrm{cT} 3 \mathrm{~b}$ cN0, tested three cycles of pembrolizumab every 3 weeks before RC. The primary end point was pCR. Of 50 enrolled patients, $54 \%$ had cT3, $42 \%$ cT2, and $4 \%$ cT2-3N1 disease. Pathological complete response was achieved in $42 \%$ of patients and downstaging to nonmuscle-invasive disease in $54 \%$. In patients with PD-L1 positive tumors, the pCR was even higher (54\%) compared to the $13.3 \%$ in patients with PD-L1 negative tumors $(n=15)$. In addition, tumor mutational burden (TMB) was associated with pCR rates [24].

The adverse events were similar to those in the metastatic trials and neither delay, nor failure to undergo RC was noted in both neoadjuvant CPI trials.

Recently, results from the NABUCCO trial have been presented at ESMO 2019. Patients with stage III urothelial cancer (cT3-4aN0M0 or cT1-4aN1-3M0) were treated with a sequence of ipilimumab and nivolumab. Primary endpoint was feasibility of resection $(<12$ weeks), secondary endpoints were pCR and safety. In this cohort of 24 patients, the preoperative combination CPI regimen, resulted in a pCR of 46 and $60 \%$ for allcomers and patients with PDL1 positive tumors, respectively. A downstaging to non-muscle invasive disease was observed in $73 \%$ patients with PD-L1 positive tumors [26]. Despite showing very promising results with similar or even higher pCR rates compared to historic data of conventional cisplatin-based NAC, these studies require confirmation in larger randomized phase III trials. Of special interest are ongoing clinical trials evaluating the effectiveness of neoadjuvant application of novel combinations of chemotherapy with CPI and targeting agents.

\section{Timing of surgery}

Due to unavailable prospective data, there is uncertainty regarding the optimal timing of RC. However, RC should be performed as soon as possible following a patient's completion of and recovery from NAC. In multivariate analyses by Alva et al., performance of RC within 10 weeks after NAC did not compromise patient survival and, thus, provided a reasonable window for patient recovery and surgical intervention [27].

\section{Predictive biomarkers for neoadjuvant therapy}

Urothelial cancer is a highly heterogeneous disease which results in unsatisfactory response rates when therapies are given in unselected patient populations. For several years the aim was to develop predictive biomarkers to improve response rates to systemic therapy in the neoadjuvant and metastatic setting-but, unfortunately, none of them has been validated in high-quality studies [28].

Focused on molecular subtypes, potential therapeutic pathways have been proposed based on genetic signatures derived from data such as "The Cancer Genome Atlas" (TCGA) project. The analysis suggests that luminal-infiltrated and the subgroup basal/ squamous tumors might have best response to platinum-based NAC, while others may benefit more from targeted therapies (i.e., FGFR inhibitors) or CPI [29]. Retrospective analysis of independent patient cohorts revealed that patients with basal tumors have a superior outcome compared to other subtypes when neoadjuvant therapy for MIBC was given with the best response to cisplatin-based NAC [30].

A randomized phase 2 trial presented at ASCO 2019, reported on the predictive value of regimen-specific genetic alterations based on co-expression extrapolation (COXEN) models. Eligible patients were randomized to receive either GC or ddMVAC. Although, each of the COXEN scores failed to prove its regimenspecific predictive value, the GC COXEN score was a significant predictor of response independent of the NAC regimen. The planned biomarker analysis from this trial will hopefully help us better define genetic alterations that could predict response to NAC [31].

In terms of neoadjuvant CPI, PD-L1 seems to maintain a value as predictive biomarker. Indeed, results from phase 2 trials demonstrate higher pCR rates in patients with PD-L1 positive tumors compared to PDL1 negative tumors [24, 32].

Funding Open access funding provided by Medical University of Vienna.

Conflict of interest K.M. Gust has received advisory board fees from Cepheid, Roche, MSD, and Ferring; speaker fees from Astellas, AstraZeneca, BMS, Ipsen, Janssen, MSD, and Roche; and meeting/travel expenses from Allergan, Astellas, AstraZeneca, Bayer, BMS, Janssen, MSD, Novartis, Pfizer, Pierre Fabre, and Roche. S.F. Shariat has received advisory board and/or speaker fees from Astellas, AstraZeneca, Bayer, BMS, Cepheid, Ferring, Ipsen, Janssen, Lilly, MSD, Olympus, Pfizer, Pierre Fabre, Roche, Sanochemia, Sanofi, and Wolff. S. Brönimann and D. D'Andrea declare that they have no competing interests.

Open Access This article is distributed under the terms of the Creative Commons Attribution 4.0 International License (http://creativecommons.org/licenses/by/4.0/), which permits unrestricted use, distribution, and reproduction in any medium, provided you give appropriate credit to the original author(s) and the source, provide a link to the Creative Commons license, and indicate if changes were made. 


\section{References}

1. Hautmann RE, de Petriconi RC, Pfeiffer C, Volkmer BG. Radical cystectomy for urothelial carcinoma of the bladder withoutneoadjuvantoradjuvant therapy: long-termresults in 1100 patients. Eur Urol. 2012;61(5):1039-47.

2. Stein JP, Skinner DG. Radical cystectomy for invasive bladder cancer: long-term results of a standard procedure. World J Urol. 2006;24(3):296-304.

3. Kluth LA, Black PC, Bochner BH, Catto J, Lerner SP, Stenzl A, et al. Prognostic and prediction tools in bladder cancer: a comprehensive review of the literature. Eur Urol. 2015;68(2):238-53.

4. Svatek RS, Shariat SF, Novara G, Skinner EC, Fradet Y, Bastian PJ, et al. Discrepancy between clinical and pathological stage: external validation of the impact on prognosis in an international radical cystectomy cohort. BJU Int. 2011;107(6):898-904.

5. Abdollah F, Gandaglia G, Thuret R, Schmitges J, Tian Z, Jeldres $\mathrm{C}$, et al. Incidence, survival and mortality rates of stage-specific bladder cancer in United States: a trend analysis. Cancer Epidemiol. 2013;37(3):219-25.

6. Advanced Bladder Cancer Meta-analysis Collaboration. Neoadjuvant chemotherapy in invasive bladder cancer: a systematic review and meta-analysis. Lancet. 2003;361(9373):1927-34.

7. Advanced Bladder Cancer Meta-analysis Collaboration. Neoadjuvant chemotherapy in invasive bladder cancer: update of a systematic review and meta-analysis of individual patient data. Eur Urol. 2005;48(2):202-5. discussion $5-6$.

8. Zargar H, Espiritu PN, Fairey AS, Mertens LS, Dinney CP, Mir MC, et al. Multicenter assessment of neoadjuvant chemotherapy for muscle-invasive bladder cancer. Eur Urol. 2015;67(2):241-9.

9. Galsky MD, Pal SK, Chowdhury S, Harshman LC, Crabb SJ, Wong YN, et al. Comparative effectiveness of gemcitabine plus cisplatin versus methotrexate, vinblastine, doxorubicin, plus cisplatin as neoadjuvant therapy for muscleinvasive bladder cancer. Cancer. 2015;121(15):2586-93.

10. Grossman HB, Natale RB, Tangen CM, Speights VO, Vogelzang NJ, Trump DL, et al. Neoadjuvant chemotherapy plus cystectomy compared with cystectomy alone for locally advanced bladder cancer. $\mathrm{N}$ Engl J Med. 2003;349(9):859-66.

11. Choueiri TK, Jacobus S, Bellmunt J, Qu A, Appleman LJ, Tretter C, et al. Neoadjuvant dose-dense methotrexate, vinblastine, doxorubicin, and cisplatin with pegfilgrastim support in muscle-invasive urothelial cancer: pathologic, radiologic, and biomarker correlates. J Clin Oncol. 2014;32(18):1889-94.

12. Sternberg CN, de Mulder PH, Schornagel JH, Theodore C, Fossa SD, van Oosterom AT, et al. Randomized phase III trial of high-dose-intensity methotrexate, vinblastine, doxorubicin, and cisplatin (MVAC) chemotherapy and recombinant human granulocyte colony-stimulating factor versus classic MVAC in advanced urothelial tract tumors: European Organization for Research and Treatment ofCancer Protocol no. 30924. JClin Oncol. 2001;19(10):2638-46.

13. Peyton CC, Tang D, Reich RR, Azizi M, Chipollini J, PowSang JM, et al. Downstaging and survival outcomes associated with neoadjuvant chemotherapy regimens among patients treated with cystectomy for muscle-invasive bladder cancer. JAMAOncol. 2018;4(11):1535-42.

14. Yin M, Joshi M, Meijer RP, Glantz M, Holder S, Harvey HA, et al. Neoadjuvant chemotherapy for muscle-invasive bladder cancer: a systematic review and two-step metaanalysis. Oncologist. 2016;21(6):708-15.

15. Hanna N, Trinh QD, Seisen T, Vetterlein MW, Sammon J, Preston MA, et al. Effectiveness of neoadjuvant chemotherapy for muscle-invasive bladder cancer in the current real world setting in the USA. Eur Urol Oncol. 2018;1(1):83-90.

16. Azizi M, Cheriyan SK, Peyton CC, Foerster B, Shariat SF, Spiess PE. Optimal management of upper tract urothelial carcinoma: an unmet need. Curr Treat Options Oncol. 2019;20(5):40.

17. Matin SF, Margulis V, Kamat A, Wood CG, Grossman HB, Brown GA, et al. Incidence of downstaging and complete remission after neoadjuvant chemotherapy for highrisk upper tract transitional cell carcinoma. Cancer. 2010;116(13):3127-34.

18. HosogoeS, HatakeyamaS, Kusaka A, Hamano I, Iwamura H, Fujita N, et al. Platinum-based neoadjuvant chemotherapy improves oncological outcomes in patients with locally advanced upper tract urothelial carcinoma. Eur Urol Focus. 2018;4(6):946-53.

19. Kubota Y, Hatakeyama S, Tanaka T, Fujita N, Iwamura H, Mikami J, et al. Oncological outcomes of neoadjuvant chemotherapyin patients withlocally advancedupper tract urothelial carcinoma: a multicenter study. Oncotarget. 2017;8(60):101500-8.

20. Porten S, Siefker-Radtke AO, Xiao L, Margulis V, Kamat AM, Wood CG, et al. Neoadjuvant chemotherapy improves survival of patients with upper tract urothelial carcinoma. Cancer. 2014;120(12):1794-9.

21. Gust KM, Shariat SF. Re: high response rates to neoadjuvant chemotherapy in high-grade upper tract urothelial carcinoma. Practice update. 2019. https://www. practiceupdate.com/content/high-response-rates-toneoadjuvant-chemotherapy-in-high-grade-upper-tracturothelial-carcinoma/83023. Accessed 31 May2019.

22. Necchi A, Raggi D, Gust K, D’Andrea D, Briganti A, Capitanio U, et al. PURE-02: an open label, multicenter, single-arm, phase 2 study of neoadjuvant pembrolizumab (Pembro), preceding radical nephroureterectomy (RNU), for patients with localized high-risk urothelial carcinoma of the upper urinary tract (UTUC). Eur Urol Suppl. 2019;18(1):e1835.

23. Gust KM, Shariat SF. Re: Pembrolizumab as neoadjuvant therapy before radical cystectomy in patients with muscle-invasive urothelial bladder carcinoma (PURE-01): an open-label, single-arm, phase II study. Eur Urol. 2019;75(4):695-6.

24. NecchiA, AnichiniA, RaggiD, BrigantiA, MassaS, Luciano R, etal. Pembrolizumab as neoadjuvant therapybefore radical cystectomy in patients with muscle-invasive urothelial bladder carcinoma (PURE-01): an open-label, single-arm, phase II study. JClin Oncol. 2018;:JCO1801148. https:// doi. org/10.1200/JCO.18.01148

25. Castellano D, Duran MartinezI, Rodríguez-VidaA, CrabbSJ, van der Heijden MS, Font Pous A, et al. A phase II study investigating the safety and efficacy of neoadjuvent atezolizumab in muscle invasive bladder cancer (ABACUS). Ann Oncol. 2018;29(suppl_8). https://doi.org/10.1093/ annonc/mdy283.108

26. Van der Heijden MS, Dijk N, Smit L, et al. 904PD Preoperative ipilimumab and nivolumab in locoregionally advanced, stage III, urothelial cancer (NABUCCO). Ann Oncol. 2019;30(Suppl5). https://doi.org/10.1093/annonc/ mdz249.003

27. Alva AS, Tallman CT, He C, Hussain MH, Hafez K, Montie JE, et al. Efficient delivery of radical cystectomy after neoad- 
juvant chemotherapy for muscle-invasive bladder cancer: a multidisciplinary approach. Cancer. 2012;118(1):44-53.

28. Shariat SF, Lotan Y, Vickers A, Karakiewicz PI, SchmitzDrager BJ, Goebell PJ, et al. Statistical consideration for clinical biomarker research in bladder cancer. Urol Oncol. 2010;28(4):389-400.

29. Robertson AG, Kim J, Al-Ahmadie H, Bellmunt J, Guo G, Cherniack $\mathrm{AD}$, et al. Comprehensive molecular characterization of muscle-invasive bladder. Cancer Cell. 2018;174(4):1033.

30. Seiler R, Ashab HAD, Erho N, van Rhijn BWG, Winters B, Douglas J, et al. Impact of molecular subtypes in muscle-invasive bladder cancer on predicting response and survival after neoadjuvant chemotherapy. Eur Urol. 2017;72(4):544-54.

31. Flaig TW, Tangen CM, Daneshmand S, Shivaram Alva A, Lerner SP, Lucia MS, et al. SWOG S1314: a randomized phase II study of co-expression extrapolation (COXEN) with neoadjuvant chemotherapy for localized, muscle-invasive bladder cancer. J Clin Oncol. 2019;37. https://doi.org/10. 1200/JCO.2019.37.15_suppl.4506

32. Powles T, Rodriguez-Vida A, Duran I, Crabb SJ, Van Der Heijden MS, Pous AF, et al. A phase II study investigating the safety and efficacy of neoadjuvant atezolizumab in muscle invasive bladder cancer (ABACUS). J Clin Oncol. 2018;36(15_suppl):4506.

Publisher's Note Springer Nature remains neutral with regard to jurisdictional claims in published maps and institu-

tional affiliations.

- For latest news from international oncology congresses see: http://www.springermedizin.at/ memo-inoncology 